\title{
Fluorescent Sensors Based on Cu-Doped Carbon Quantum Dots for the Detection of Rutin
}

\author{
Cheng-ya Wang, ${ }^{a}$ Shanzhai Shang, ${ }^{a}$ Xudong Zheng, ${ }^{a}$ Ping Lei, ${ }^{a}$ Jingmei Han, ${ }^{a}$ Linda Yuan, ${ }^{a}$ \\ Zhiqiang Li, ${ }^{a}$ Ru Wang, ${ }^{a}$ Weimin Gong, ${ }^{a}$ Jianguo Tang $*, a$ and Yaling Yang ${ }^{\circledR *, b}$ \\ ${ }^{a}$ China Tobacco Yunnan Industrial Co., Ltd, 650231 Kunming, China \\ ${ }^{b}$ Faculty of Life Science and Technology, Kunming University of Science and Technology, \\ 650500 Kunming, China
}

\begin{abstract}
In this paper, $\mathrm{Cu}$-doped carbon quantum dots ( $\mathrm{Cu}-\mathrm{CQDS})$ were prepared by carrying out thermolysis of $\mathrm{Na}_{2}[\mathrm{Cu}(\mathrm{EDTA})]$ and hydroxylamine hydrochloride at $300{ }^{\circ} \mathrm{C}$ for $2 \mathrm{~h}$. The asprepared $\mathrm{Cu}-\mathrm{CQD}$ s were characterized by UV-Vis, Fourier transform infrared (FTIR) spectroscopy, transmission electron microscopy (TEM), X-ray photoelectron spectroscopy (XPS) and X-ray diffraction (XRD). The Cu-CQDs exhibited good fluorescence property, high stability, excitationdependent emission behavior and a quantum yield of $9.8 \%$. More significantly, the synthesized $\mathrm{Cu}-\mathrm{CQD}$ s were developed for sensing rutin (RT) based on the fluorescence quenching of the prepared $\mathrm{Cu}-\mathrm{CQDs}$. A good linear relationship between the fluorescence quenching of $\mathrm{Cu}-\mathrm{CQDS}$ and different concentrations of RT was obtained in the range of $0.1-15 \mu \mathrm{g} \mathrm{mL}^{-1}$ with a correlation coefficient of 0.9959 . The detection limit was as low as $0.05 \mu \mathrm{g} \mathrm{mL}^{-1}$ (signal-to-noise ratio, $\mathrm{S} / \mathrm{N}=3$ ). The possible mechanism of fluorescence quenching was explored, which was proved to be inner filter effect. Importantly, the proposed method was successfully applied in the detection of RT tablet samples and with satisfactory results, showing the practical applications.
\end{abstract}

Keywords: $\mathrm{Cu}$-doped carbon quantum dots, rutin, $\mathrm{Na}_{2}[\mathrm{Cu}(\mathrm{EDTA})]$, fluorescence sensors

\section{Introduction}

As a novel fluorescent carbon nanomaterial with size below $10 \mathrm{~nm},{ }^{1,2}$ carbon quantum dots (CQDs) have attracted more and more attention in recent years owing to low cost, green synthesis, good biosafety, environmental friendliness as well as excellent optical properties such as excitation-dependent emissions behavior and tunable photoluminescence (PL). ${ }^{3-8}$ CQDs provided an exciting opportunity as alternative to heavy metal (e.g., $\mathrm{Hg}, \mathrm{Cd}, \mathrm{Pb}$ )based semiconductor quantum dots,, 910 and were applied in biological imaging, ${ }^{11,12}$ fluorescent sensors ${ }^{13-15}$ and catalysis. ${ }^{16}$ The optical and fluorescence properties of CQDs arise from quantum confinement and edge effects, and also depend upon functional groups on the surface of CQDs. ${ }^{17}$ More interestingly, due to the modulation in the band structure, metal atom doping can not only ameliorate the optical properties but also increase the electron donating/ accepting abilities, and the surface charges of CQDs could be changed. ${ }^{18-21}$ Ethylenediaminetetraacetic acid (EDTA) is

*e-mail: jgtang@163.com; yilyil8@163.com one of common starting materials for CQDs, which with a saturated Schiff-base-like structure could form EDTA chelate such as $\left(\mathrm{Na}_{2}[\mathrm{Cu}(\mathrm{EDTA})]\right)$, and the $\mathrm{Na}_{2}[\mathrm{Cu}($ EDTA) pyrolyzed to form a flaky graphite conductive structure CQDs. ${ }^{22}$

Rutin (RT) (quercetin-3-ehamnosylglucoside, Figure 1) is a kind of flavonoid glycoside. ${ }^{23}$ Many studies have revealed that rutin is good for human health, such as antibacterial, antiulcer, anti-inflammatory, antiarrhythmia, antitumor, protective and expanding blood vessel..$^{24,25} \mathrm{Up}$ to now, lots of analytical methods have been applied for the<smiles>CC1OC(OCC2OC(Oc3c(-c4ccc(O)c(O)c4)oc4cc(O)cc(O)c4c3=O)C(O)C(O)C2O)C(O)C(O)C1O</smiles>

Figure 1. The molecular structure of rutin. 
determination of rutin, including chemiluminescence, ${ }^{26,27}$ high-performance liquid chromatography (HPLC), ${ }^{28,29}$ capillary electrophoresis spectrophotometry, ${ }^{30,31} \mathrm{UV}$-Vis spectrophotometry ${ }^{32}$ and electrochemical methods. ${ }^{33-36}$

In this work, $\mathrm{Cu}$-doped carbon quantum dots (Cu-CQDs) were prepared by a facile one-step pyrolytic synthesis using $\mathrm{Na}_{2}[\mathrm{Cu}($ EDTA) $]$ as precursor. The saturated Schiff-base-like structure transforms into a $\mathrm{Cu}$ coordination complex chelated with graphene matrices during pyrolysis, which is confirmed by Fourier transform infrared (FTIR) spectroscopy, transmission electron microscopy (TEM), $\mathrm{X}$-ray diffraction (XRD) and X-ray photoelectron spectroscopy (XPS). The formed Cu-CQDs were bluish green luminescence under $365 \mathrm{~nm}$ and showed emission maximum at $412 \mathrm{~nm}$ when excited at $354 \mathrm{~nm}$. With the addition of rutin, the emission intensity at $412 \mathrm{~nm}$ of $\mathrm{Cu}-\mathrm{CQDs}$ decreased. Based on the phenomenon, the method was established to determinate concentration of rutin.

\section{Experimental}

\section{Materials and apparatus}

$\mathrm{Na}_{2}[\mathrm{Cu}(\mathrm{EDTA})]$, baicalin, chlorogenic acid, quercetin and hydroxylamine hydrochloride were all provided by Aladdin Chemistry (Shanghai, China). Rutin, $\mathrm{Fe}\left(\mathrm{NO}_{3}\right)_{3}$, $\mathrm{Hg}(\mathrm{Ac})_{2}, \mathrm{MgCl}_{2}, \mathrm{Mn}\left(\mathrm{NO}_{3}\right)_{2}, \mathrm{~Pb}\left(\mathrm{NO}_{3}\right)_{2}, \mathrm{Zn}\left(\mathrm{NO}_{3}\right)_{2}$ and $\mathrm{CdCl}_{2}$ were purchased from Damao Chemical Corp (Tianjin, China). Stock solution of $1.0 \mathrm{mg} \mathrm{mL}^{-1}$ rutin was prepared in methanol and stored below $4{ }^{\circ} \mathrm{C}$. Rutin tablets (Shanxi Yunpeng pharmaceutical Co. Ltd., B080302) and compound rutin tablets (Shanghai Zhaohui pharmaceutical Co. Ltd., 090904) were used as samples. All of the reagents were used without further purification. Ultra-pure water was used during the all experiment.

Ultraviolet-visible (UV-Vis) absorption spectrum was performed on a UV-2550 spectrophotometer (Shimadzu, Kyoto, Japan). The fluorescence spectrum was recorded using molecular fluorescence spectrometer (Agilent Technologies, California, USA). The morphologies of the samples were characterized using a transmission electron microscope (Philips-FEI, Eindhoven, Holland). FTIR spectroscopy was performed on a TENSOR27 FTIR spectrometer (Bruker, Karlsruhe, Germany). XPS analyses were carried out on an X-ray photoelectron spectrometer (Kratos, Manchester, UK). XRD was recorded using a D8-advance X-ray diffractometer (Bruker, Karlsruhe, Germany), and a vortex mixer (Hanuo Instrument Co., Ltd., XH-B, Shanghai, China) was used in the experiment.

\section{Synthesis of Cu-CQDs}

$3 \mathrm{~g}$ of $\mathrm{Na}_{2}[\mathrm{Cu}(\mathrm{EDTA})]$ and $2 \mathrm{~g}$ of hydroxylamine hydrochloride were put into the center of a quartz tube, and calcined at $300{ }^{\circ} \mathrm{C}$ for $2 \mathrm{~h}$ at a heating rate of $5{ }^{\circ} \mathrm{C} \mathrm{min}^{-1}$ under $\mathrm{N}_{2}$ atmosphere. Then the product was ground and dissolved in $100 \mathrm{~mL}$ water, and the suspension was treated with ultrasound $(300 \mathrm{~W}, 40 \mathrm{kHz})$ for $15 \mathrm{~min}$ at room temperature. After centrifugation for $20 \mathrm{~min}$ at $10000 \mathrm{rpm}$, the upper brown solution was filtered with $0.22 \mu \mathrm{m}$ membrane to remove the non-fluorescent deposited $\mathrm{Na}$-salts. The solution was dialyzed with MD34 (3500 Da) dialysis tube for $48 \mathrm{~h}$ to remove the remaining salts and small fragments. The concentrated solution was dried at $60{ }^{\circ} \mathrm{C}$ for $24 \mathrm{~h}$, and $\mathrm{Cu}-\mathrm{CQD}$ s powder was obtained. The amount of carbon dots obtained after purification was about 0.4-0.5 g.

\section{Measure of fluorescence quantum yield}

In general, quantum yield (QY) is measured by a standard fluorophore solution which shows a well-known quantum yield (Q). The QY of the Cu-CQDs was measured using quinine sulfate (QY of $54 \%$ at $360 \mathrm{~nm}, \eta=1.33$ ) as a reference, which was dissolved in a $0.1 \mathrm{~mol} \mathrm{~L}^{-1} \mathrm{H}_{2} \mathrm{SO}_{4}$ aqueous solution. The QY of the as-prepared Cu-CQDs was calculated according to equation 1 :

$\mathrm{Q}=\mathrm{Q}_{\mathrm{R}} \times \frac{\mathrm{I}_{\mathrm{S}} \mathrm{A}_{\mathrm{R}} \eta_{\mathrm{S}}^{2}}{\mathrm{I}_{\mathrm{R}} \mathrm{A}_{\mathrm{S}} \eta_{\mathrm{R}}^{2}}$

where Q represents QY, A represents the absorbance at the excitation wavelength, I represents the integrated emission intensity, $\eta$ represents the solvent refraction index. The subscript ' $S$ ' and ' $R$ ' refer to the samples and quinine sulfate, respectively.

\section{Rutin tablet samples pretreatment}

10 pieces of rutin tablets were ground to powder in a mortar, and $10 \mathrm{mg}$ of the powder was dissolved in $25 \mathrm{~mL}$ of ethanol. Recovery experiments were performed by adding different concentrations of rutin to the treated samples.

\section{Results and Discussion}

\section{Characterization of Cu-CQDs}

The optical properties of the Cu-CQD were investigated by UV-Vis absorption and fluorescence emission spectra. The UV-Vis spectrum (Figure 2a) showed two shoulders at 
269 and $321 \mathrm{~nm}$. The absorption at $269 \mathrm{~nm}$ corresponded to $\pi^{*}-\pi^{*}$ of $\mathrm{C}=\mathrm{C} / \mathrm{C}=\mathrm{N}$ and the absorption at $321 \mathrm{~nm}$ was ascribed to $\mathrm{n}-\pi *$ transition of the $\mathrm{C}=\mathrm{O} / \mathrm{C}-\mathrm{NH}_{2}$ bond. ${ }^{2} \mathrm{In}$ addition, the color of the $\mathrm{Cu}-\mathrm{CQD}$ solution was brown, and exhibited strong blue fluorescence (Figure 2a inset) when excited with a UV lamp (365 nm). As it can be seen in Figure 2b, the maximum emission wavelength of the $\mathrm{Cu}$-CQDs solution was located at $412 \mathrm{~nm}$ with the excitation wavelength of $354 \mathrm{~nm}$. Therefore, $354 \mathrm{~nm}$ was fixed as the optimal excitation wavelength for all the fluorescence measurements. Figure $2 c$ exhibited the relationship between excitation and emission wavelength at room temperature. When excited Cu-CQDs by different wavelength at 314, 324, 334, 344, 354, 364, 374, 384 and $394 \mathrm{~nm}$, the corresponding photoluminescence (PL) emission peak located at 398, 400, 404, 410, 412, 416, 426, 438 and $446 \mathrm{~nm}$, respectively. Obviously, the emission wavelength was red-shifted with gradual lowering of PL intensity and with the increasing excitation wavelength. This excitation wavelength-dependent PL behavior could be contributed to the surface state of Cu-CQDs, which affected the band structure, and this phenomenon can be resulted by the different sizes, complex $\mathrm{Cu}$ doping or diverse surface emissive trap sites. ${ }^{37}$ Moreover, the fluorescent quantum yield (QY) of the Cu-CQDs in aqueous solution was measured to be ca. 9.8\% using quinine sulfate as reference. The QY of the Cu-CQDs is higher when compared with the result that was reported in other literatures for carbon dots..$^{38-40}$

As shown in Figure 3a, TEM image clearly revealed the uniform morphology and narrow size distribution of the synthesized Cu-CQDs. The prepared Cu-CQDs exhibited good dispersion and were uniform in shape with an average diameter of $4 \mathrm{~nm}$. The high-resolution TEM image (Figure 3a, inset) revealed the crystallinity of $\mathrm{Cu}$ CQDs with a lattice parameter of ca. $0.21 \mathrm{~nm}$, which is consistent with the basal spacing of graphite. Obviously, its dispersion and uniformity were in agreement with the fact that it actually dissolves. As shown in Figure 3b, XRD of the $\mathrm{Cu}-\mathrm{CQDs}$ showed a strong peak at around $2 \theta=27.7^{\circ}$, which corresponds to the graphitic structure. ${ }^{41}$ The thermal stability of synthesized Cu-CQDs was analyzed by thermogravimetry analysis (TGA), which was shown in Figure 3c. The TGA of Cu-CQDs showed a $\mathrm{H}_{2} \mathrm{O}$ evaporation weight loss below $100^{\circ} \mathrm{C}$ and other sharp weight loss at around $350-600{ }^{\circ} \mathrm{C}$, which is presumably due to the loss of $\mathrm{CO}$ and $\mathrm{CO}_{2}$ from the oxygen-containing functional groups. Figure $3 \mathrm{~d}$ showed the FTIR spectrum of the as-prepared Cu-CQDs. The broad band in the region of $3100-3500 \mathrm{~cm}^{-1}$ was assigned to stretching vibrations of $\mathrm{C}-\mathrm{H}$ and $\mathrm{N}-\mathrm{H}$. The peak at $2939 \mathrm{~cm}^{-1}$ could be identified as the stretching vibrations of $\mathrm{C}-\mathrm{H}$. The peaks at 1662 ,
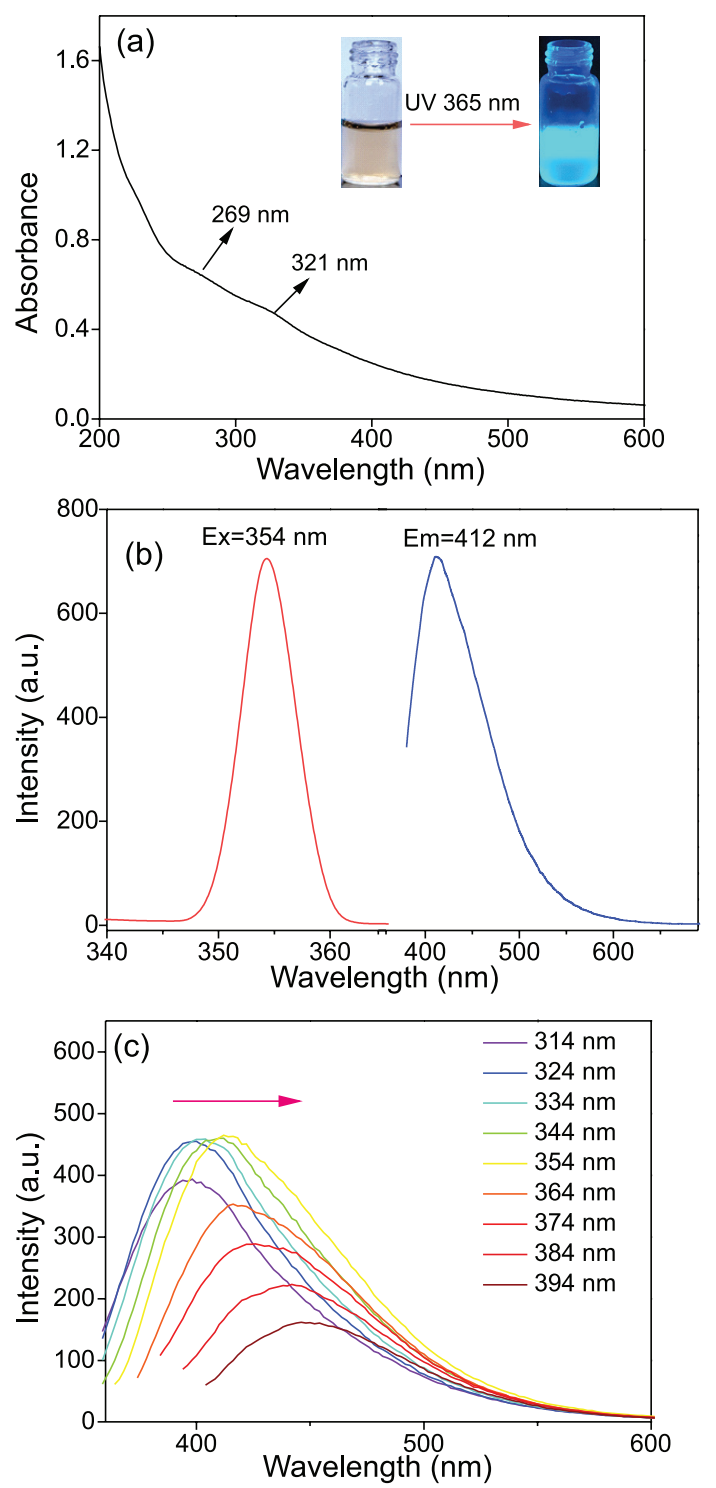

Figure 2. (a) UV-Vis absorption spectra (abs), inset: photographs of the solution of the Cu-CQDs taken under visible light (left) and $365 \mathrm{~nm} \mathrm{UV}$ light (right); (b) fluorescence excitation $\left(\lambda_{\mathrm{ex}}\right)$ and emission $\left(\boldsymbol{\lambda}_{\mathrm{em}}\right)$ spectra of the $\mathrm{Cu}-\mathrm{CQD}$; ; (c) fluorescence emission spectra of $\mathrm{Cu}-\mathrm{CQD}$ s at different excitation wavelengths from 314 to $394 \mathrm{~nm}$ in $10 \mathrm{~nm}$ increments.

1404 and $1296 \mathrm{~cm}^{-1}$ were attributed to the stretching vibrations of $\mathrm{C}=\mathrm{O}, \mathrm{C}-\mathrm{N}$ and $\mathrm{C}-\mathrm{O}$, respectively. The full XPS scan of Cu-CQDs was shown in Figure 3e, indicating that the synthesized $\mathrm{Cu}-\mathrm{CQD}$ s contains $\mathrm{C}, \mathrm{O}, \mathrm{N}$ and $\mathrm{Cu}$ elements with the content ratios of 46.31, 27.14, 4.26 and $0.98 \%$, respectively. In detail, Figure $3 \mathrm{f}$ shows the high resolution $\mathrm{C} 1 \mathrm{~s}$ spectrum of $\mathrm{Cu}-\mathrm{CQDs}$, the three peaks at $284.5,285.7$ and $287.6 \mathrm{eV}$ could be assigned to $\mathrm{C}=\mathrm{C}$, $\mathrm{C}-\mathrm{N}$ and $\mathrm{C}=\mathrm{O}$. Figure $3 \mathrm{~g}$ shows the high resolution $\mathrm{O} 1 \mathrm{~s}$ spectrum of $\mathrm{Cu}-\mathrm{CQD}$, the three main peaks at 530.5, 531.7 and $535.2 \mathrm{eV}$ could be attributed to $\mathrm{C}=\mathrm{O}, \mathrm{C}-\mathrm{O}-\mathrm{C}$ and $\mathrm{C}-\mathrm{O}-\mathrm{H}$. The detailed high resolution $\mathrm{Cu} 2 \mathrm{p}$ spectrum (Figure $3 \mathrm{~h}$ ) shows the existence of $\mathrm{Cu}$ in $\mathrm{Cu}$-doped CQDs 
(932.3, 933.3, 952.4 eV) corresponding to the spin orbit splitting of $\mathrm{Cu} 2 \mathrm{p} 3 / 2$ and $\mathrm{Cu} 2 \mathrm{p} 1 / 2$, respectively, indicating the presence of $\mathrm{Cu}^{\mathrm{II}}$ and $\mathrm{Cu}^{0}$ in as-prepared
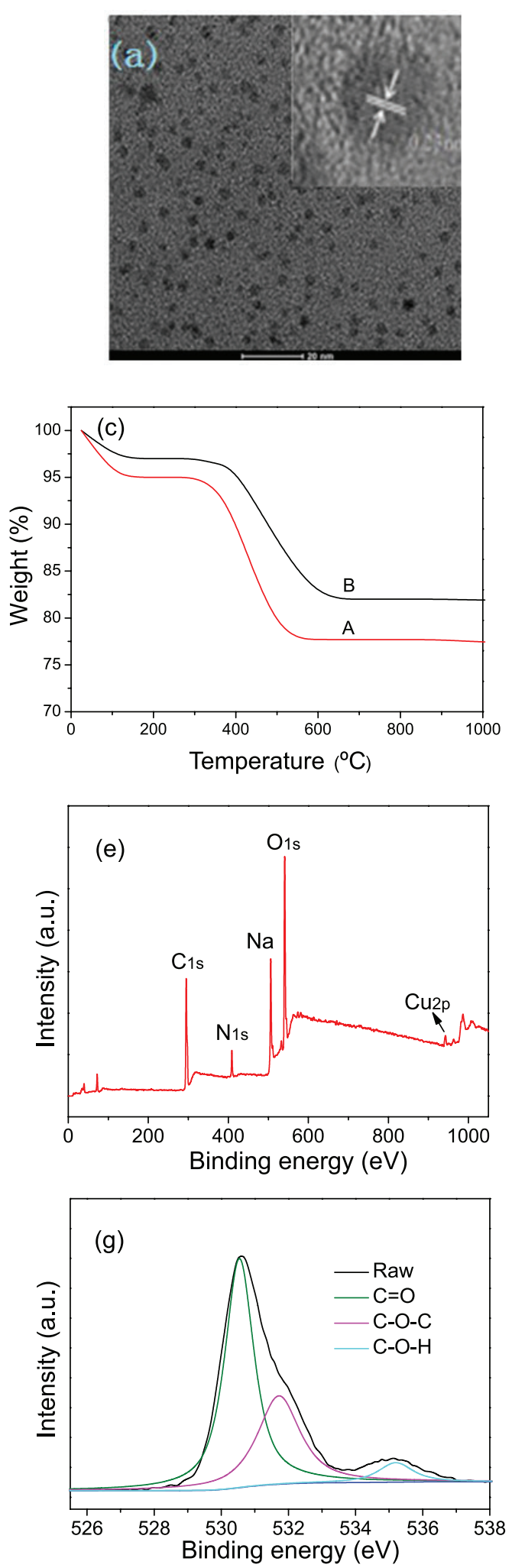

Cu-doped CQDs. ${ }^{42,43}$ The results from XPS analysis were in good agreement with FTIR. XPS and FTIR data demonstrated that the surface of $\mathrm{Cu}-\mathrm{CQD}$ s were rich in
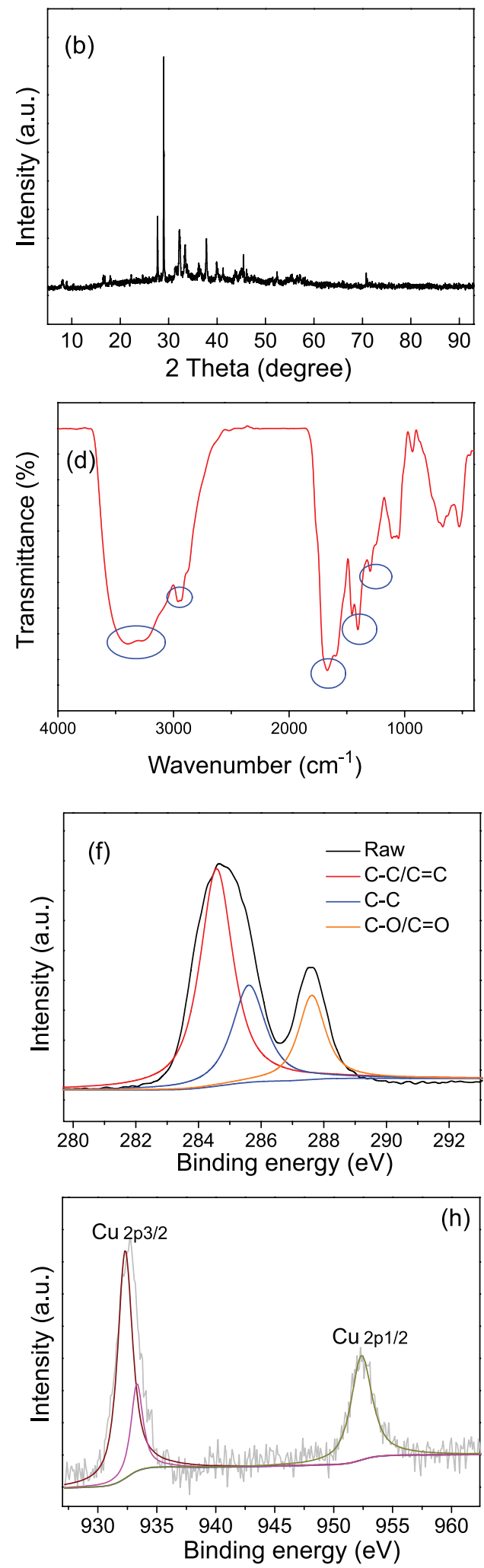

Figure 3. (a) TEM image; (b) XRD pattern; (c) TGA curves of CQDs (A) and Cu-CQDs (B); (d) FTIR spectrum; (e) full-scan XPS spectrum of the $\mathrm{Cu}-\mathrm{CQDs}$; (f-h) high-resolution $\mathrm{C} 1 \mathrm{~s}, \mathrm{O} 1 \mathrm{~s}$ and $\mathrm{Cu} 2 \mathrm{p}$ XPS spectrum of the synthesized Cu-CQDs. 
water-soluble groups, such as hydroxyl groups, amino groups and carboxyl groups.

\section{Mechanism of $\mathrm{Cu}-\mathrm{CQDs}$ quenching}

Inner filter effect (IFE) would occur when the absorption spectrum of quencher in the detection system overlapped with the excitation or emission spectra of CQDs. Generally, highly effective IFE requires that the absorption band of the absorbent should overlap sufficiently with the excitation of the fluorophore and/or the emission band. Therefore, it is important to choose an appropriate absorber and fluorophore pair in the IFE-based fluorescence nano-probe. ${ }^{44}$ Figure 4a showed the UV absorption spectrum of RT. It could be observed that there were two wide absorption peaks at 265 and $362 \mathrm{~nm}$. The as-prepared Cu-CQDs were chosen as the fluorophore because of the large overlap between the excitation and emission spectra of the prepared Cu-CQDs and UV absorption of RT. The overlap would cause RT to shield excitation and emission light from Cu-CQDs. Therefore, the fluorescence of $\mathrm{Cu}-\mathrm{CQD}$ could be successfully quenched by the absorbance enhancement of RT, which assures that the IFE occurs in a highly efficient way. Furthermore, fluorescence lifetime was considered to explore the possible mechanism of RT quenching the fluorescence of Cu-CQDs. As shown in Figure $4 b$, the average fluorescence lifetime of Cu-CQDs almost had no significant change after the addition of RT. The results indicated that the main possible mechanism is IFE. ${ }^{45}$ Besides, the essentially unchanged fluorescence lifetime of the system before and after the addition of RT also indicated that the fluorescence quenching is static quenching. ${ }^{46}$ The possible mechanism is shown in Scheme 1.

\section{Optimum condition of sensitivity for the RT sensor}

\section{Effect of $\mathrm{pH}$ and ionic strength}

To evaluate the stability of the Cu-CQDs, the effects of $\mathrm{pH}$ and ionic strength on the fluorescence intensity were
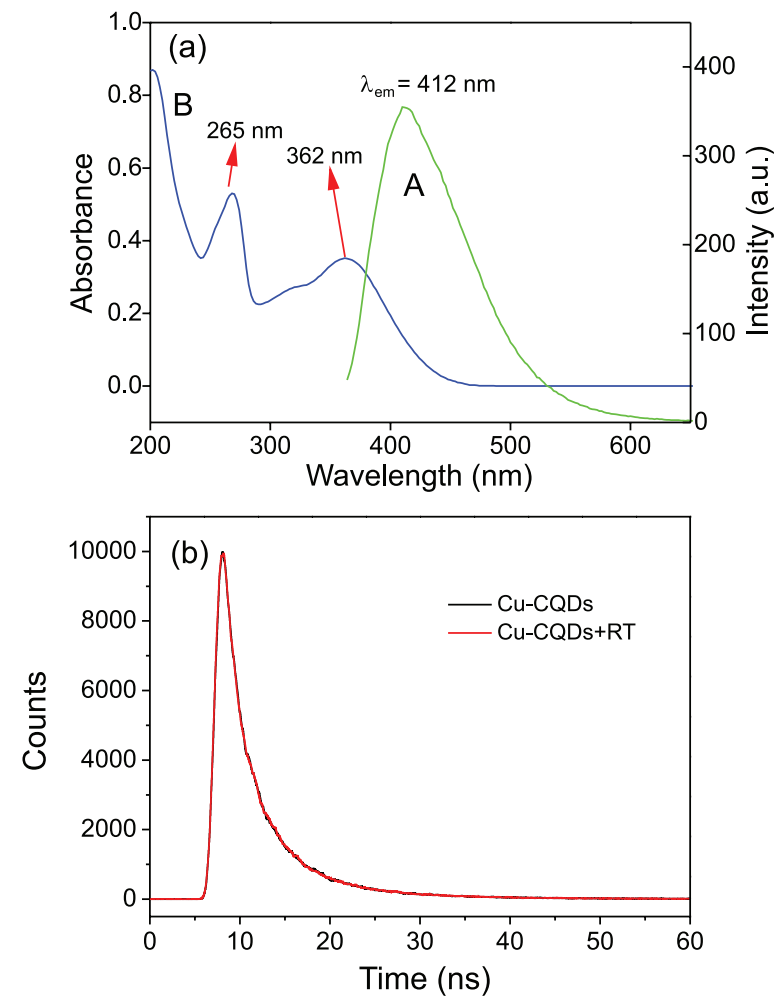

Figure 4. (a) The absorption (abs: A) of RT and emission ( $\lambda_{\mathrm{em}}$ : B) spectra of the Cu-CQDs; (b) fluorescence emission decay curve of $\mathrm{Cu}-\mathrm{CQDs}$ and $\mathrm{Cu}-\mathrm{CQDs}+\mathrm{RT}$.

further examined. As shown in Figure S1a (Supplementary Information (SI) section), the fluorescence intensity was obtained under different $\mathrm{pH}$ conditions. As the results suggested, the intensity of fluorescence of the prepared $\mathrm{Cu}-\mathrm{CQDs}$ was increasing as the $\mathrm{pH}$ value rose from 2.0 to 5.0. Then the intensity was almost unaltered with the change of $\mathrm{pH}$ values in the range of 5.0-8.0. The $\mathrm{pH}$ owned a small impact on the fluorescence of prepared $\mathrm{Cu}$-CQDs under weak acidic or alkaline condition, which may be due to the protonation or de-protonation of the functional groups on the surface of Cu-CQDs. Accordingly, it was demonstrated that the structure of $\mathrm{Cu}-\mathrm{CQDs}$ was relatively stable and not simply ruined within the $\mathrm{pH}$ range of 5.0-8.0.

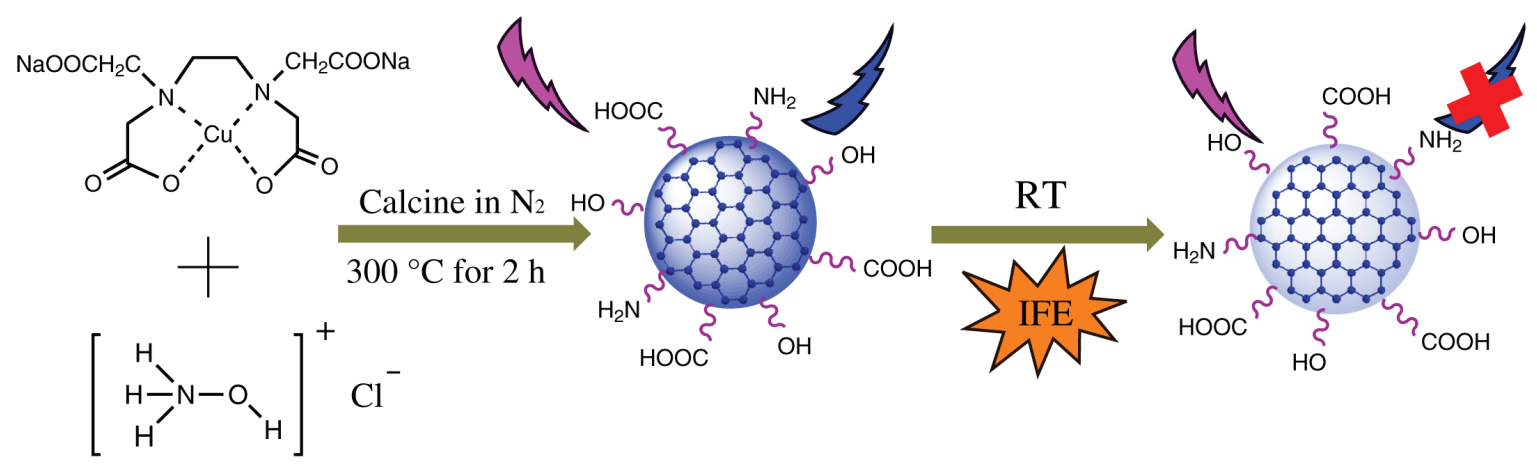

Scheme 1. Schematic illustration of the sensitive fluorescence detection of RT based on Cu-CQDs. 
We also have investigated the influence of ionic strength on the fluorescence of the Cu-CQDs. As shown in Figure S1b (SI section), different concentrations of $\mathrm{NaCl}$ solutions range from 0 to $2 \mathrm{~mol} \mathrm{~L}^{-1}$ were added into the $\mathrm{Cu}$-CQDs. The fluorescence intensity maintained almost unchanged with the increasing $\mathrm{NaCl}$ concentrations, implying high stability of the $\mathrm{Cu}-\mathrm{CQDs}$ even under high ionic strength environment. The stability of the effective fluorescence is conducive to the application of the Cu-CQDs.

\section{Stability of Cu-CQDs for the detection of RT}

Stability is an important point for $\mathrm{Cu}-\mathrm{CQD}$ s to detect the RT. To confirm the fluorescence intensity of Cu-CQDs in the process of detecting RT, different incubation time was investigated. As shown in Figure 5a, the fluorescence intensity of $\mathrm{Cu}-\mathrm{CQD}$ s decreased after the addition of RT, and maintained basically unchanged after 2 min. In addition, the fluorescence intensity could remain unchanged for $30 \mathrm{~min}$. These results showed that the proposed method was fast and stable for the detection of RT. Therefore, the reaction time for the RT determination was set to $2 \mathrm{~min}$. Moreover, Figure $5 \mathrm{~b}$ showed the long stability of the as-prepared Cu-CQDs. It can be observed that the $\mathrm{Cu}-\mathrm{CQDs}$ had good long stability. The Cu-CQDs still exhibited strong fluorescence intensity after 3 months
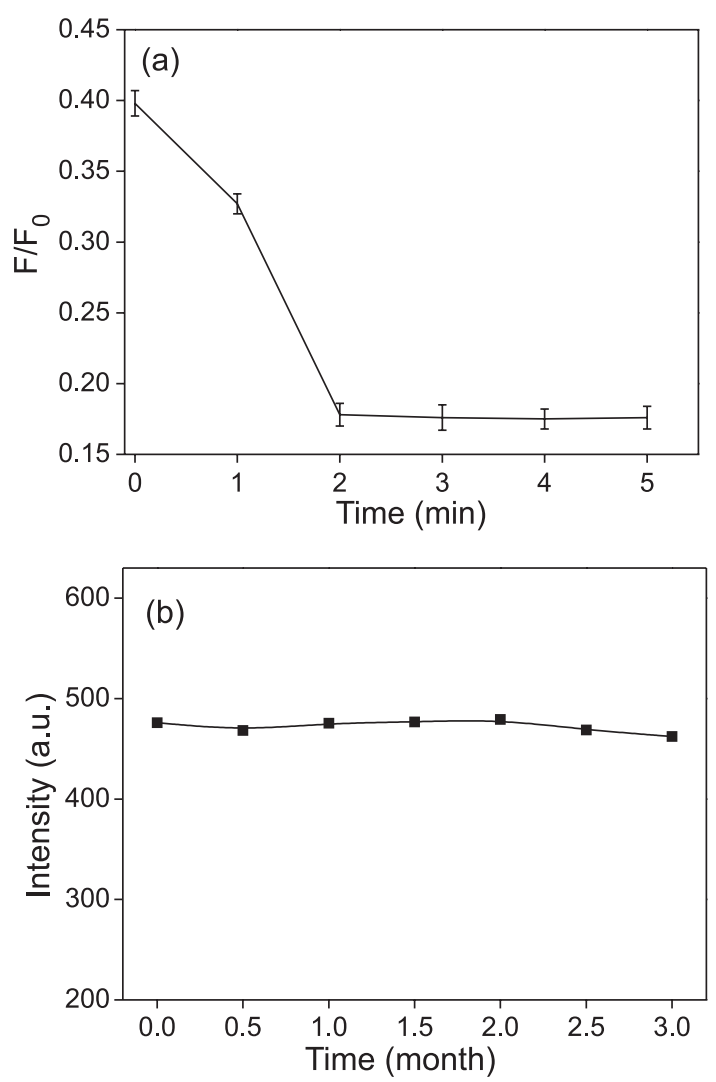

Figure 5. (a) Fluorescence intensity of Cu-CQDs with RT at different reaction time and (b) fluorescence intensity of $\mathrm{Cu}-\mathrm{CQDs}$ storage times. storage at $4^{\circ} \mathrm{C}$. Meanwhile, Cu-CQDs could be re-dispersed in water for using after being dried to powders in vacuum oven. The result showed that the fluorescence intensity had no obvious decrease.

\section{Selectivity of RT detection}

To evaluate the selectivity of Cu-CQDs as a fluorescent probe for the detection of RT, the fluorescence intensity of $\mathrm{Cu}$-CQDs was examined in the presence of different possible interfering substances, including urea, $\mathrm{Fe}\left(\mathrm{NO}_{3}\right)_{3}$, $\mathrm{FeSO}_{4}, \mathrm{Hg}(\mathrm{Ac})_{2}, \mathrm{MgCl}_{2}, \mathrm{Mn}\left(\mathrm{NO}_{3}\right)_{2}, \mathrm{~Pb}\left(\mathrm{NO}_{3}\right)_{2}, \mathrm{CdCl}_{2}$, $\mathrm{Zn}\left(\mathrm{NO}_{3}\right)_{2}$, baicalin, chlorogenic acid and quercetin. As shown in Figure 6, RT and quercetin could efficiently quench the fluorescence intensity of the Cu-CQDs, while the influence of the other possible interfering substances on the fluorescence intensity of the Cu-CQDs was negligible. However, the interference of quercetin against rutin would be avoided in the practical rutin detection in tablets samples. In following studies, the analytical capability of this fluorescence probe would be evaluated in rutin tablets without the potential coexistence of quercetin. As discussed in the characterization of prepared $\mathrm{Cu}-\mathrm{CQDS}$, the surface of the as-prepared $\mathrm{Cu}$-CQDs was rich in hydroxyl groups, amino groups and carboxyl groups. The selectivity of Cu-CQDs towards RT is mainly due to the hydrogen bonding between the hydroxyl groups of RT and amine, amide and carboxyl groups of Cu-CQDs in addition to $\pi-\pi$ interaction of the aromatic stack of Cu-CQDs with RT.

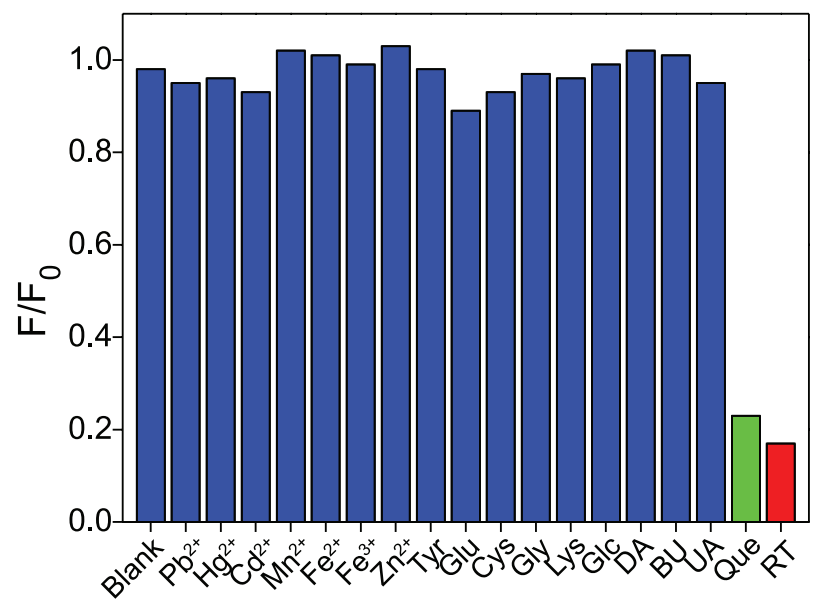

Figure 6. Selectivity of the Cu-CQDs to different possible interfering substances.

\section{Sensitivity of RT detection}

To estimate the sensitivity of Cu-CQDs toward RT, the fluorescence spectra were recorded with the addition of different concentrations of RT. Figure 7a showed 
that the fluorescence intensity centered at $412 \mathrm{~nm}$ of $\mathrm{Cu}-\mathrm{CQD}$ decreased gradually with the addition of various concentration of RT. It is discussed previously that this fluorescent quenching of Cu-CQDs after the addition of RT is owing to the inner filter effect (IFE). That is to say, the absorption of RT shields the excitation or emission wavelengths of $\mathrm{Cu}-\mathrm{CQDs}$, and lead to the fluorescence quenching. Additionally, this trend is in agreement with the fluorescence quenching photographs (insets in Figure 7b), in which the color of the solution gradually changes from bright blue to dark blue under the same conditions. A good linear relationship was obtained between the fluorescence quenching and different concentration of RT in the range of 0.1 to $15 \mu \mathrm{g} \mathrm{mL}^{-1}$ with a regression equation of $\mathrm{F} / \mathrm{F}_{0}=0.059 \mathrm{C}+0.0283$ (correlation coefficient $\left(R^{2}\right)=0.9959$ ) (insets in Figure $7 a$ ), where $F_{0}$ and $F$ represent the fluorescent intensities of $\mathrm{Cu}-\mathrm{CQDs}$ in the absence and presence of RT, and C is the different concentration of RT. The detection limit of RT is estimated to be $0.05 \mu \mathrm{g} \mathrm{mL}^{-1}$ at a signal-to-noise ratio of $3(\mathrm{~S} / \mathrm{N}=3)$. The $\mathrm{Cu}-\mathrm{CQDs}$ prepared in the work showed a relatively lower limit of detection when compared with other reported literatures which detected RT (Table 1).

\section{Determination of RT in drug samples}

To assess the practicality of the method, medicinal rutin tablets were chosen as real sample, which was ground by a mortar and dissolved in absolute ethanol to get the sample solutions. The sample solutions were diluted 50 times before using. Then the sample solutions were measured according to the as-proposed method with the data shown in Table 2. The recoveries were in the range of $97.5-102.5 \%$ and relative standard deviation (RSD) values were below $2.21 \%$. The results indicated the reliable and efficient application of $\mathrm{Cu}-\mathrm{CQDs}$ as fluorescent sensors for rutin determination.
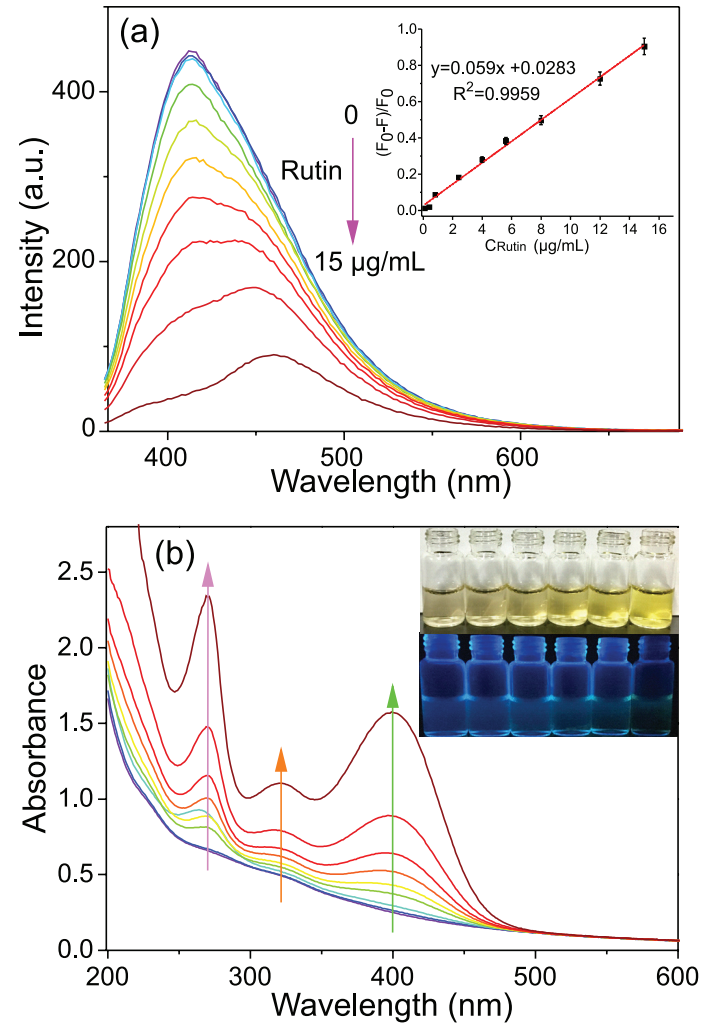

Figure 7. (a) Fluorescence emission spectra of $\mathrm{Cu}-\mathrm{CQD}$ s by treating with various RT concentrations $\left(0.1-15 \mu \mathrm{g} \mathrm{mL}^{-1}\right)$ (inset: the relationship between the $\left(\mathrm{F}_{0}-\mathrm{F}\right) / \mathrm{F}_{0}$ and the RT concentrations) and (b) absorption spectra with various RT concentrations (inset: photographs under ambient daylight (top) and UV light (bottom)).

\section{Conclusions}

In summary, highly fluorescent $\mathrm{Cu}-\mathrm{CQDs}$ were synthesized by a green and low cost route from $\mathrm{Na}_{2}[\mathrm{Cu}(\mathrm{EDTA})]$ and hydroxylamine hydrochloride. The prepared $\mathrm{Cu}-\mathrm{CQDs}$ showed good fluorescence properties and water solubility. Based on the phenomenon that the fluorescence intensity of Cu-CQDs could be selectively

Table 1. Comparison of different methods for the detection of RT

\begin{tabular}{lccc}
\hline Method & Linear range $\left./(\mu \mathrm{g} \mathrm{mL})^{-1}\right)$ & Limit of detection $\left./(\mu \mathrm{g} \mathrm{mL})^{-1}\right)$ & Reference \\
\hline Fluorescence & $0.31-9.15$ & 0.06 & 47 \\
Electrochemistry & $0.14-1.53 \times 10^{3}$ & 0.07 & 48 \\
Sequential injection analysis & $0.3-24$ & 0.13 & 49 \\
Fluorescence & $0.1-15$ & 0.05 & this work \\
\hline
\end{tabular}

Table 2. Determination of RT in real samples $(n=3)$

\begin{tabular}{lcccccc}
\hline Sample & Found $/\left(\mu \mathrm{g} \mathrm{mL}^{-1}\right)$ & Added $/\left(\mu \mathrm{gL}^{-1}\right)$ & Total $/(\mu \mathrm{g} \mathrm{mL}-1)$ & $\mathrm{RSD}(\mathrm{n}=3) / \%$ & Recovery $/ \%$ & HPLC $\left./(\mu \mathrm{g} \mathrm{mL})^{-1}\right)$ \\
\hline B080302 & 7.9 & 4.0 & 11.9 & 1.80 & 102.5 & 11.8 \\
090904 & 8.0 & 4.0 & 12.0 & 2.21 & 97.5 & 12.0 \\
\hline
\end{tabular}

RSD: relative standard deviation; HPLC: high-performance liquid chromatography. 
quenched by rutin, the $\mathrm{Cu}-\mathrm{CQD}$ s were used as nano-sensors for the sensitive and selective detection of RT. This novel proposed method provided good linear RT detection in the range of $0.1-15 \mu \mathrm{g} \mathrm{mL}{ }^{-1}$ with a detection limit of $0.05 \mu \mathrm{g} \mathrm{mL} \mathrm{L}^{-1}$. The possible mechanism of fluorescence quenching was discussed, which was proved to be inner filter effect and static quenching. The successful application of the Cu-CQDs in the detection of RT in pharmaceutical products demonstrated the proposed method have great application prospect.

\section{Supplementary Information}

Supplementary information (the effects of $\mathrm{pH}$ and ionic strength on the fluorescence intensity of Cu-CQDs) is available free of charge at http://jbcs.sbq.org.br as PDF file.

\section{Acknowledgments}

The work was strongly supported by school-enterprise cooperation project (2018530101000312).

\section{References}

1. Baker, S. D.; Baker, G. A.; Angew. Chem., Int. Ed. 2010, 49, 6726.

2. Li, H.; Kang, Z.; Liu, Y.; Lee, S.-T.; J. Mater. Chem. 2012, 22, 24230.

3. Lim, S. Y.; Shen, W.; Gao, Z.; Chem. Soc. Rev. 2015, 44, 362.

4. Zhu, S.; Meng, Q.; Wang, L.; Zhang, J.; Song, Y.; Jin, H.; Zhang, K.; Sun, H.; Wang, H.; Yang, B.; Angew. Chem., Int. Ed. 2013, 52,3953

5. Na, N.; Ma, Z.; Fei, H.; Li, S.; Jian, L.; Liu, S. X.; Yang, P.; Langmuir 2017, 33, 5786.

6. Shen, J.; Zhu, Y.; Yang, X.; Li, C.; ChemInform 2012, 43, 3686.

7. Ray, S. C.; Saha, A.; Jana, N. R.; Sarkar, R.; J. Phys. Chem. C 2009, 113, 18546.

8. Tang, L.; Ji, R.; Cao, X.; Lin, J.; Jiang, H.; Li, X.; Teng, K. S.; Luk, C. M.; Zeng, S.; Hao, J.; ACS Nano 2012, 6, 5102.

9. Deng, J.; Lu, Q.; Mi, N.; Li, H.; Liu, M.; Xu, M.; Tan, L.; Xie, Q.; Zhang, Y.; Yao, S.; Chem. - Eur. J. 2014, 20, 4993.

10. Huang, X.; Zhang, F.; Zhu, L.; Choi, K. Y.; Guo, N.; Guo, J.; Tackett, K.; Anilkumar, P.; Liu, G.; Quan, Q.; ACS Nano 2013, $7,5684$.

11. Chen, J.; Liu, J.; Li, J.; Xu, L.; Qiao, Y.; J. Colloid Interface Sci. 2017, 485, 167.

12. Edison, T. N.; Atchudan, R.; Sethuraman, M. G.; Shim, J. J.; Lee, Y. R.; J. Photochem. Photobiol., B 2016, 161, 154.

13. Wang, N.; Wang, Y.; Guo, T.; Yang, T.; Chen, M.; Wang, J.; Biosens. Bioelectron. 2016, 85, 68.

14. Chen, D.; Xu, M.; Wu, W.; Li, S.; J. Alloys Compd. 2017, 701, 75.
15. Wu, X.; Song, Y.; Yan, X.; Zhu, C.; Ma, Y.; Du, D.; Lin, Y.; Biosens. Bioelectron. 2017, 94, 292.

16. Liu, R.; Huang, H.; Li, H.; Liu, Y.; Zhong, J.; Li, Y.; Zhang, S.; Kang, Z.; ACS Catal. 2014, 4, 328.

17. Ying, Y. L.; Peng, X. S.; Analyst 2016, 141, 2619.

18. Martindale, B. C.; Hutton, G. A.; Caputo, C. A.; Reisner, E.; J. Am. Chem. Soc. 2015, 137, 6018.

19. Bourlinos, A. B.; Bakandritsos, A.; Kouloumpis, A.; Gournis, D.; Krysmann, M.; Giannelis, E. P.; Polakova, K.; Safarova, K.; Hola, K.; Zboril, R.; J. Mater. Chem. 2012, 22, 23327.

20. Xu, Q.; Liu, Y.; Su, R.; Cai, L.; Li, B.; Zhang, Y.; Zhang, L.; Wang, Y.; Wang, Y.; Li, N.; Nanoscale 2016, 8, 17919.

21. Wu, W.; Zhan, L.; Fan, W.; Song, J.; Li, X.; Li, Z.; Wang, R.; Zhang, J.; Zheng, J.; Wu, M.; Angew. Chem., Int. Ed. 2015, 127, 6640.

22. Reddy, G. R.; Balasubramanian, S.; Chennakesavulu, K.; J. Mater. Chem. A 2014, 2, 15598.

23. Li, X.; Zhang, Y.; Yuan, Z.; Chromatographia 2002, 55, 243.

24. Magdalena, J. S.; Agnieszka, Z. G.; Food Anal. Methods 2014, 7, 2033.

25. Muti, M.; Gençdağ, K.; Nacak, F. M.; Aslan, A.; Colloids Surf., B 2013, 106, 181.

26. Song, Z.; Hou, S.; Talanta 2002, 57, 59.

27. Li, S.; Zhang, L.; Chen, L.; Zhong, Y.; Ni, Y.; Anal. Methods 2016, 8, 4056.

28. Zhang, X.; Zhou, X.; Liu, X.; Li, X.; Whang, W.; J. Chromatogr. Sci. 2015, 54, 574.

29. Doshi, G. M.; Zine, S. P.; Chaskar, P. K.; Une, H. D.; J. Sep. Sci. 2014, 6, 234.

30. Wang, W.; Lin, P.; Ma, L.; Xu, K.; Lin, X.; J. Sep. Sci. 2016, 39, 1357.

31. Altaweel, A. M.; Abdelkader, M. S.; Fawzy, G. A.; Perveen, S.; Maher, H. M.; Alzoman, N. Z.; Alshehri, M. M.; Aljohar, H.; Alshowiman, H.; Pak. J. Pharm. Sci. 2015, 28, 1897.

32. Hao, X.; Ying, L.; Tang, H. W.; Liu, C. M.; Wu, Q. S.; Anal. Lett. 2010, 43, 893.

33. Li, S.; Yang, B.; Wang, C.; Wang, J.; Feng, Y.; Yan, B.; Xiong, Z.; Du, Y.; J. Electroanal. Chem. 2017, 786, 20.

34. Gholivand, M. B.; Mohammadibehzad, L.; Hosseinkhani, H.; Anal. Biochem. 2015, 493, 35.

35. Arvand, M.; Shabani, A.; Ardaki, M. S.; Food Anal. Methods 2017, 10, 2332.

36. Yang, X.; Long, J.; Sun, D.; Electroanalysis 2016, $28,83$.

37. Cui, X.; Wang, Y.; Liu, J.; Yang, Q.; Zhang, B.; Gao, Y.; Wang, Y.; Lu, G.; Sens. Actuators, B 2016, 242, 1272.

38. Lu, W.; Qin, X.; Liu, S.; Chang, G.; Zhang, Y.; Luo, Y.; Asiri, A.; Al-Youbi, A.; Sun X.; Anal. Chem. 2012, 84, 5351.

39. Zhou, J.; Sheng, Z.; Han, H.; Zou, M.; Li, C.; Mater. Lett. 2012 , 66, 222.

40. De, B.; Karak, N.; RSC Adv. 2013, 3, 8286. 
41. Wang, J.; Sahu, S.; Sonkar, S. K.; Ii, K. N. T.; Sun, K. W.; Liu, Y.; Maimaiti, H.; Anilkumar, P.; Sun, Y. P.; RSC Adv. 2013, 3, 15604.

42. Gayathri, P.; Kumar, A. S.; Langmuir 2014, 30, 10513.

43. Röckert, M.; Franke, M.; Tariq, Q.; Ditze, S.; Stark, M.; Uffinger, P.; Wechsler, D.; Singh, U.; Xiao, J.; Marbach, H.; Steinrück, H.-P.; Lytken, O.; Chem. - Eur. J. 2014, $20,8948$.

44. Zhang, D.; Dong, Z.; Jiang, X.; Feng, M.; Li, W.; Gao, G.; Anal. Methods 2013, 5, 1669.

45. Yang, H.; Yang, L.; Yuan, Y.; Pan, S.; Yang, J.; Yan, J.; Zhang, H.; Sun, Q.; Hu, X.; Spectrochim. Acta, Part A 2017, 189, 139.
46. Li, N.; Liu, S. G.; Fan, Y. Z.; Ju, Y. J.; Xiao, N.; Luo, H. Q.; Li, N. B.; Anal. Chim. Acta 2018, 1013, 63.

47. Sinduja, B.; Abraham, J. S.; Spectrochim. Acta, Part A 2018 , 193, 486

48. Rohani, T.; Ghaderi, A.; J. Anal. Chem. 2018, 73, 277.

49. Al-Shwaiyat, M. K. E. A.; Miekh, Y. V.; Denisenko, T. A.; Vishnikin, A. B.; Andruch, V.; Bazel, Y. R.; J. Pharm. Biomed. Anal. 2017, 149, 179.

Submitted: July 6, 2018

Published online: December 14, 2018 